\title{
Interval Observer-based Feedback Control for Rehabilitation in Tremor
}

\author{
Kwassi H. Degue, Denis Efimov and Jerome Le Ny
}

\begin{abstract}
Tremor, one of the most common health disorders, is defined as a chronic movement disorder. To reduce tremor in patients, the design of stabilizing techniques is critical. For this purpose, we consider an uncertain continuoustime linear time-varying oscillator model of tremor in this article. We design a state feedback control for deep brain stimulation technique by considering the practical case in which only sets of admissible values are given for the nominal values of the stimulation amplitude, the time-varying tremor's angular frequency and the tremor measurement noise. First, we estimate state signal bounds that include the true unknown value of the state. Next, we design a stabilizing control input based on the estimated bounds. The stability of the controlled system is verified using linear matrix inequalities (LMIs). We demonstrate the methodology's performance in numerical simulations.
\end{abstract}

\section{INTRODUCTION}

Tremor, which is an involuntary, approximately sinusoidal movement and roughly rhythmic, is one of the most common types of movement disorders [1]. Indeed, 6.38 to 7.63 million people were living with Essential Tremor in the USA in 2014 [2]. Tremor has impacts on sundry parts of the body, but the hands are the most affected. It is not a life-threatening disease, but it usually seriously lessens the patients' quality of life, since they are not able to execute simple daily tasks such as opening a door or drinking a glass of water [3].

An effective therapy for pathological tremor is Deep Brain Stimulation (DBS) [4]-[6]. Namely, in Parkinson disease patients, one surgically implants electrodes in the basal ganglia, commonly in the subthalamic nucleus, to generate electric-pulse stimulation of multifarious neurons [7], [8]. Some characteristics of the high-frequency electronic pulse-train that is applied to the brain are pulse-

This work was supported in part by NSERC under Grant RGPIN5287-2018 and RGPAS-2018-522686, by the Pierre Arbour Foundation doctoral scholarship, a FRQNT doctoral scholarship, the Government of Russian Federation (Grant 08-08) and the Ministry of Science and Higher Education of Russian Federation (passport of goszadanie no. 2019-0898).

K. H. Degue and J. Le Ny are with the department of Electrical Engineering, Polytechnique Montreal and GERAD, QC H3T-1J4, Montreal, Canada. kwassi-holali.degue, jerome. le-ny@polymt l.ca

D. Efimov is with Inria, Univ. Lille, CNRS, UMR 9189 - CRIStAL, F-59000 Lille, France and ITMO University, 197101 Saint Petersburg, Russia. Denis.Efimoveinria.fr width, amplitude and frequency. Over the last decade, advanced techniques of controlled DBS have been proposed to decrease tremor in patients [9]-[12]. Feedback control techniques have been proposed in [10]-[12] by assuming that one can measure the perfect value of the tremor, which constitutes a drawback since real measurements are usually corrupted by noise. An output feedback control technique has been developed in [9] by assuming that an estimation of the perfect value of the stimulation amplitude is possible, which can be a limitation in many cases. Indeed, it has been shown that an estimation of such a perfect value can be highly inaccurate in several cases [9]. In addition, [9] assumes noiseless outputs in their model, i.e., they assume that the noise in the tremor signal is totally removed by means of a band-pass filter. Nevertheless, for example by using a second-order Butterworth filter from 3 to $10 \mathrm{~Hz}$ in case of tremor [13], [14], only noisy measurements are usually available. Moreover, they propose a static output feedback controller, which does not include an observer. When considering dynamic systems, a state feedback control technique has better performances than a static output feedback controller. Furthermore, when one has to handle the presence of exogenous disturbances or uncertain parameters, whose values are only known to belong to an interval or polytope, all the aforementioned methodologies that are proposed in the existing literature (based on point-wise estimation) cannot be applied. The use of methodologies based on interval estimators [15]-[17] instead of point-wise observers can address such problems.

The contribution of this article consists in designing for the first time a stabilizing state feedback control based on an interval observer methodology to reduce tremor in patients. We consider an uncertain continuous-time linear time-varying oscillator model of tremor, with four types of uncertainty: the initial condition, the nominal value of the stimulation amplitude, the time-varying tremor's angular frequency and the measurement noise. For these parameters, we assume that the exact values are not available, only bounds are given. Following [18], [19], first, we evaluate the state signal bounds, assuming that the control signal is given. Next, we use these bounds to design a stabilizing control law for the observer's system. The stabilization of 
the state bounds around a vicinity of zero implies the same property for the state due to the inclusion relation. Note that only noiseless systems have been considered in [18] when applying such a methodology. Furthermore, we consider a stabilizing control design in this article, in contrast to [20][23] that has studied only observer design problems.

We present the problem statement in Section II. Then, we rewrite the model as a linear parameter-varying (LPV) system and review some results on the theory of interval estimation and on positive systems in Section III. We apply these results to design state signal bounds and a stabilizing control input in the rest of Section III. Finally, we illustrate the performance of our methodology by presenting numerical simulations to reduce the tremor of a Parkinson's disease patient.

Notation. We denote the real numbers by $\mathbb{R}$, the integers by $\mathbb{Z}, \mathbb{R}_{+}=\{\tau \in \mathbb{R}: \tau \geq 0\}$ and $\mathbb{Z}_{+}=\mathbb{Z} \cap \mathbb{R}_{+}$. We denote the cones of vectors of dimension $n$ with positive, nonnegative, and negative components by $\mathbb{R}_{>0}^{n}, \mathbb{R}_{+}^{n}$ and $\mathbb{R}_{<0}^{n}$ respectively. For a vector $x \in \mathbb{R}^{n}$, we denote $|x|_{\infty}:=$ $\max _{i \in\{1, \ldots, n\}}\left|x_{i}\right|,|x|=|x|_{2}:=\left(\sum_{i=1}^{k}\left|x_{i}\right|^{2}\right)^{1 / 2}$ and we denote the induced 2-norm of a matrix $A \in \mathbb{R}^{n \times n}$ by $|A|$. For a vector-valued signal $u: \mathbb{R}_{+} \rightarrow \mathbb{R}^{n}$, we denote its

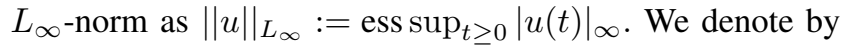
$\mathcal{L}_{\infty}^{n}$ the set of signals $u$ with the property $\|u\|_{L_{\infty}}<\infty$. The $n \times n$ identity matrix is denoted $I_{n}$ and the matrix with all elements equal to 0 and dimension $p \times 1$ is denoted $0_{p}$. For two vectors $x_{1}, x_{2} \in \mathbb{R}^{n}$ or matrices $A_{1}, A_{2} \in \mathbb{R}^{n \times n}$, the relations $x_{1} \leq x_{2}$ and $A_{1} \leq A_{2}$ are understood elementwise. A matrix $A \in \mathbb{R}^{n \times n}$ is Hurwitz if all its eigenvalues have negative real parts; it is called Metzler if all its elements outside of the main diagonal are nonnegative. The notation $P \prec 0(P \succ 0)$ means that the matrix $P \in \mathbb{R}^{n \times n}$ is symmetric and negative (positive) definite.

\section{BACKGROUND}

One can characterize a DBS signal by several parameters, including but not limited to, frequency $\omega$ and stimulation amplitude $a$ that are supplied to a stimulus generator in order to create a stimulus signal [9]. One applies such a stimulus signal to a particular part of the brain by using an electrode in order to overcome cognitive disorder's motor symptoms. One can quantify the tremor that is caused by a brain disorder in terms of position, velocity and acceleration, by using a sensor that is fixed to a patient's finger [14]. One can attenuate the noise in the tremor signal by using a band-pass filter, for example a secondorder Butterworth filter from 3 to $10 \mathrm{~Hz}$ [13], [14]. Further information about the tremor measurement's process can be found in [9], [14].

The whole process dynamics can be modeled as a simple time-varying oscillator, where DBS parameters are considered as inputs and the filtered tremor signal as the output. Throughout this article, we consider the following continuous-time linear time-varying oscillator model of tremor dynamics [9], whose design has been inspired by [24]

$$
\begin{aligned}
& \dot{x}_{1}(t)=-\omega^{2}(t) x_{2}(t)+u(t)-a \\
& \dot{x}_{2}(t)=x_{1}(t),
\end{aligned}
$$

where $x:=\left[\begin{array}{ll}x_{1} & x_{2}\end{array}\right]^{\mathrm{T}} \in \mathbb{R}^{2}$ is the state vector: $x_{1}$ represents the normalized tremor (normalized with the strength of amplitude [9]) and $x_{2}$ is a state variable of the oscillator, $u \in \mathbb{R}$ stands for the control input, $\omega \in \mathbb{R}$ represents the time-varying tremor's angular frequency and $a$ is the stimulation amplitude's nominal value for which the tremor related to a brain disorder vanishes. The presence of the parameter $a$ in the model (1) is appropriate since in case of brain disease such as Parkinson, the tremor of a patient is controlled by DBS after selecting stimulation parameters [25]-[27]. As in [9], we assume that the parameter $\omega(t)$ is uncertain but two bounds $\underline{\omega}, \bar{\omega} \in \mathbb{R}_{+}$are known such that $\underline{\omega} \leq \omega(t) \leq \bar{\omega}, \forall t \geq 0$. In contrast with [9], which have assumed that an estimation of the perfect value of the parameter $a$ is possible, we assume in this article that $a$ is unknown but two bounds $\underline{a}, \bar{a} \in \mathbb{R}$ are given such that $\underline{a} \leq a \leq \bar{a}, \forall t \geq 0$. Indeed, it has been proved that an estimation of a perfect value of $a$ can be highly inaccurate in several cases [9].

Furthermore, in contrast to [10]-[12], where it is assumed that the tremor is measured perfectly, we consider the practical case in which measurements consist of the tremor corrupted by noise as follows

$$
y(t)=x_{1}(t)+v(t),
$$

where $v \in \mathcal{L}_{\infty}$ represents a measurement noise. We assume that $v$ is unknown but two bounds $\underline{v}, \bar{v} \in \mathbb{R}$ are given such that $\underline{v} \leq v(t) \leq \bar{v}, \forall t \geq 0$. The presence of such a measurement noise is natural since by using a band-pass filter, one has access to a noisy measurement of the tremor signal only.

In this article, we aim at reducing the tremor caused by a brain disorder such that $x_{1}$ tracks a vicinity of a desired (healthy) trajectory $x_{1}^{d}=0$. Hence, the goal of this paper consists in stabilizing system (1) around 0.

\section{MAIN RESULTS}

Recall that $\omega(t)$ is an uncertain parameter that satisfies $\omega(t) \in \Theta \subset \mathbb{R}$, where the set $\Theta$ is given $(\Theta=[\underline{\omega}, \bar{\omega}])$. By using the structure of the system (1)-(2), we can rewrite it 
as follows

$$
\begin{aligned}
\dot{x}(t) & =\left(A_{0}+\Delta A(\omega(t))\right) x(t)+B u(t)-B a, \\
y_{t} & =C x(t)+v(t),
\end{aligned}
$$

with

$$
\begin{aligned}
A_{0} & =\left[\begin{array}{ll}
0 & 1 \\
1 & 0
\end{array}\right], \Delta A(\omega(t))=\left[\begin{array}{cc}
0 & -\omega^{2}(t)-1 \\
0 & 0
\end{array}\right], \\
B & =\left[\begin{array}{l}
1 \\
0
\end{array}\right], C=\left[\begin{array}{ll}
1 & 0
\end{array}\right] .
\end{aligned}
$$

We treat $a$ as an unknown input. By using the definitions of the parameter $\omega(t)$ and the set $\Theta$ and using the structure of the system (3), we deduce that the matrix $\Delta A(\omega(t))$ is uncertain but there exist two given matrices $\triangle A, \overline{\Delta A} \in$ $\mathbb{R}_{<0}^{2 \times 2}$ such that

$$
\underline{\Delta A} \leq \Delta A(\omega(t)) \leq \overline{\Delta A}
$$

for any $\theta \in \Theta$. Indeed, we get

$$
\begin{aligned}
& \underline{\Delta A}=\left[\begin{array}{cc}
0 & -\bar{\omega}^{2}-1 \\
0 & 0
\end{array}\right], \\
& \overline{\Delta A}=\left[\begin{array}{cc}
0 & -\underline{\omega}^{2}-1 \\
0 & 0
\end{array}\right] .
\end{aligned}
$$

Since, we aim at tracking a desired (healthy) trajectory $x^{d}=0$, we solve the problem in two stages. First, we design an interval observer for $x(t)$, i.e., state signal bounds $\underline{x}(t) \leq x(t) \leq \bar{x}(t), \forall t \geq 0$, assuming that the control signal $u(t)$ is given. Next, with the observer gain that is designed a priori, we use the interval observer to design a tracking control law $u(t)=f(\underline{x}(t), \bar{x}(t))$ that implies $\underline{x} \rightarrow x^{d}, \bar{x} \rightarrow x^{d}$ and keeps the relation $\underline{x}(t) \leq x(t) \leq$ $\bar{x}(t), \forall t \geq 0$. Notice that the tracking of $\underline{x}(t)$ and $\bar{x}(t)$ implies the same property for the state $x(t)$ due to the inclusion relation.

We assume that the initial condition $x(0)$ is unknown but two bounds $\underline{x}(0), \bar{x}(0)$ are given such that $\underline{x}(0) \leq x(0) \leq$ $\bar{x}(0)$. First, let us review some basic facts from the theory of interval estimation.

\section{A. Preliminaries on interval estimation and positive sys-} tems

Given a matrix $A \in \mathbb{R}^{m \times n}$, define $A^{+}=\max \{0, A\}$, $A^{-}=A^{+}-A$ (we use the same notation for vectors).

Lemma 1. [28] Let $x \in \mathbb{R}^{n}$ be a vector variable with $\underline{x} \leq x \leq \bar{x}$ for some $\underline{x}, \bar{x} \in \mathbb{R}^{n}$.

(1) If $A \in \mathbb{R}^{m \times n}$ is a constant matrix, then

$$
A^{+} \underline{x}-A^{-} \bar{x} \leq A x \leq A^{+} \bar{x}-A^{-} \underline{x} .
$$

(2) If $A \in \mathbb{R}^{m \times n}$ is a matrix variable and $\underline{A} \leq A \leq \bar{A}$ for some $\underline{A}, \bar{A} \in \mathbb{R}^{m \times n}$, then

$$
\begin{gathered}
\underline{A}^{+} \underline{x}^{+}-\bar{A}^{+} \underline{x}^{-}-\underline{A}^{-} \bar{x}^{+}+\bar{A}^{-} \bar{x}^{-} \leq A x \\
\leq \bar{A}^{+} \bar{x}^{+}-\underline{A}^{+} \bar{x}^{-}-\bar{A} \underline{x}^{+}+\underline{A}^{-} \underline{x}^{-} .
\end{gathered}
$$

Notice that when $\underline{A}, \bar{A} \in \mathbb{R}_{<0}^{m \times n}$, the inequality (6) simply reads

$$
\underline{A} \bar{x}^{+}-\bar{A} \bar{x}^{-} \leq A x \leq \bar{A} \underline{x}^{+}-\underline{A} \underline{x}^{-},
$$

since $\underline{A}=-\underline{A}^{-}, \underline{A}^{+}=0, \bar{A}=-\bar{A}^{-}$and $\bar{A}^{+}=0$ in such a case. Furthermore, when $A \in \mathbb{R}_{<0}^{m \times n}$, the inequality (5) simply becomes

$$
A \bar{x} \leq A x \leq A \underline{x},
$$

since $A=-A^{-}$and $A^{+}=0$.

Next, consider the following linear time-invariant (LTI) system

$$
\begin{aligned}
& \dot{x}=A x+B \omega(t), \omega: \mathbb{R}_{+} \rightarrow \mathbb{R}_{+}^{q}, \\
& y=C x+D \omega(t),
\end{aligned}
$$

with $x \in \mathbb{R}^{n}, y \in \mathbb{R}^{p}$ and $A \in \mathbb{R}^{n \times n}$ a Metzler matrix.

Lemma 2. [29], [30] Any solution of the LTI system (9) is element-wise nonnegative provided that $x(0) \geq 0$ and $B \in$ $\mathbb{R}_{+}^{n \times q}$. Furthermore, the output solution $y(t)$ is nonnegative if $C \in \mathbb{R}_{+}^{p \times n}$ and $D \in \mathbb{R}_{+}^{p \times q}$. A dynamical system satisfying all these restrictions is called cooperative (monotone) or nonnegative.

\section{B. Interval observer design}

There exists a matrix $L \in \mathbb{R}^{2 \times 1}$ such that the matrix $\left(A_{0}-L C\right)$ is Metzler to ensure nonnegativity for the interval observer's errors (for example $L=\left[\begin{array}{ll}0 & 0\end{array}\right]^{\mathrm{T}}$ satisfies such a condition due to the structure of the model (3)).

Define

$$
\begin{aligned}
& \underline{\alpha}(t)=\underline{\Delta A} \bar{x}^{+}(t)-\overline{\Delta A} \bar{x}^{-}(t), \\
& \bar{\alpha}(t)=\overline{\Delta A} \underline{x}^{+}(t)-\underline{\Delta A} \underline{x}^{-}(t) .
\end{aligned}
$$

By applying Lemma 1, it can be inferred that

$$
\underline{\alpha}(t) \leq \Delta A(\omega(t)) x(t) \leq \bar{\alpha}(t), \forall t \geq 0 .
$$

Since $-B \in \mathbb{R}_{<0}^{2 \times 1}$ by using the structure of the model (3), one can design an interval estimator for (3) as follows

$$
\begin{aligned}
\dot{\dot{x}}(t)= & \left(A_{0}-L C\right) \underline{x}(t)+\underline{\alpha}(t)+B u(t)+L y(t) \\
& -B \bar{a}-L^{+} \bar{v}+L^{-} \underline{v}, \\
\dot{\bar{x}}(t)= & \left(A_{0}-L C\right) \bar{x}(t)+\bar{\alpha}(t)+B u(t)+L y(t) \\
& -B \underline{a}+L^{-} \bar{v}-L^{+} \underline{v},
\end{aligned}
$$


where $\underline{x} \in \mathbb{R}^{2}$ and $\bar{x} \in \mathbb{R}^{2}$ stand respectively for the lower and the upper interval estimates for the state $x$. Notice the nonlinear structure of the interval observer (11) because of the presence of $\underline{\alpha}$ and $\bar{\alpha}$.

Theorem 1. The estimates $\underline{x}(t)$ and $\bar{x}(t)$ given by (11) yield the relations

$$
\underline{x}(t) \leq x(t) \leq \bar{x}(t), \forall t \geq 0
$$

provided that $\underline{x}(0) \leq x(0) \leq \bar{x}(0)$.

We skip the proofs of our results due to space limitations. One can compute the observer gain $L$ as solution of a linear program by using [23, Theorem 7] to ensure boundedness for the estimation errors $\bar{e}$ and $\underline{e}$. A control algorithm design is required to stabilize $\bar{x}, \underline{x}$ and $x$.

\section{Control Design}

The idea consists in stabilizing the completely known system (11) instead of (3). Indeed, when $\underline{x}$ and $\bar{x}$ converge to the desired trajectory $x^{d}, x$ follows the same property due to (12). First, we can treat the signal $y(t)$ in (11) as a state dependent disturbance.

Proposition 1. We have

$$
|y(t)| \leq|C|(|\underline{x}(t)|+|\bar{x}(t)|)+\max \{|\underline{v}|,|\bar{v}|\}, \forall t \geq 0 .
$$

Hence the output $y$ admits a linear upper bound in terms of $|\underline{x}(t)|$ and $|\bar{x}(t)|$. We aim at stabilizing the system (11), i.e., the states $\underline{x}$ and $\bar{x}$, since its stabilization implies the stabilization of the system (3). Next, let us prove that we can rewrite the dynamics (11) as a globally Lipschitz nonlinear system.

Denote $\varepsilon(t):=\left[\begin{array}{ll}\underline{x}^{\mathrm{T}}(t) & \bar{x}^{\mathrm{T}}(t)\end{array}\right]^{\mathrm{T}}$, and

$$
\begin{aligned}
& H=\left[\begin{array}{cc}
A_{0}-L C & 0 \\
0 & A_{0}-L C
\end{array}\right], \\
& \zeta=\left[\begin{array}{ll}
\zeta_{1}^{\mathrm{T}} & \zeta_{2}^{\mathrm{T}}
\end{array}\right]^{\mathrm{T}} \text {, } \\
& \zeta_{1}=(-B)^{+} \underline{a}-(-B)^{-} \bar{a}-L^{+} \bar{v}+L^{-} \underline{v} \text {, } \\
& \zeta_{2}=(-B)^{+} \bar{a}-(-B)^{-} \underline{a}+L^{-} \bar{v}-L^{+} \underline{v} \text {, } \\
& \phi(\varepsilon)=\left[\frac{\alpha}{\bar{\alpha}}\right] \text {. }
\end{aligned}
$$

In the sequel, also define

$$
\gamma_{\phi}=\left|\left[\begin{array}{cccc}
0 & 0 & -\overline{\Delta A} & \Delta A \\
-\underline{\Delta A} & \overline{\Delta A} & 0 & 0
\end{array}\right]\right| \text {. }
$$

Corollary 1. One can rewrite the dynamics (11) as follows

$$
\dot{\varepsilon}(t)=H \varepsilon(t)+\phi(\varepsilon(t))+\zeta+\left[\begin{array}{l}
B \\
B
\end{array}\right] u(t)+\left[\begin{array}{l}
L \\
L
\end{array}\right] y(t)
$$

where $\phi: \mathbb{R}^{4} \longrightarrow \mathbb{R}^{4}$ is a nonlinear function globally Lipschitz at the origin.
Proposition 1 and Corollary 1 ensure uniqueness for the solution of (11). Then, we need to stabilize the system (11) with respect to Lipschitz nonlinearities. We deduce that we can select the control input as a conventional state linear feedback

$$
\begin{aligned}
u(t) & =\underline{K} \underline{x}(t)+\bar{K} \bar{x}(t), \\
& =K \varepsilon(t),
\end{aligned}
$$

with $K=\left[\begin{array}{ll}\underline{K} & \bar{K}\end{array}\right]$, where $\underline{K}$ and $\bar{K}$ are two feedback gains to be designed. Denote $\mathcal{B}:=\left[\begin{array}{ll}B^{\mathrm{T}} & B^{\mathrm{T}}\end{array}\right]^{\mathrm{T}}$ and $\mathcal{L}:=$ $\left[\begin{array}{ll}L^{\mathrm{T}} & L^{\mathrm{T}}\end{array}\right]^{\mathrm{T}}$. After substituting (14) in (13), we get

$$
\dot{\varepsilon}(t)=(H+\mathcal{B} K) \varepsilon(t)+\phi(\varepsilon(t))+\zeta(t)+\mathcal{L} y(t) .
$$

Theorem 2. If there exist matrices $P^{T}=P \succ I_{4}, J=$ $P^{-1}, \mathcal{Q}^{T}=\mathcal{Q} \succ 0, \mathcal{R} \in \mathbb{R}^{1 \times 4}$ and a constant $\gamma>0$ such that

$$
\begin{gathered}
\mathcal{Z} \prec 0, \\
\mathcal{Z}=\left[\begin{array}{cccc}
\mathcal{W} & J & \mathcal{L} & I_{4} \\
J & J & 0 & 0 \\
\mathcal{L}^{T} & 0 & -1 & 0 \\
I_{4} & 0 & 0 & -\gamma I_{4}
\end{array}\right], \\
\mathcal{W}= \\
\quad J H^{T}+\mathcal{R}^{T} \mathcal{B}^{T}+H J+\mathcal{B R} \\
+\left(\gamma_{\phi}+2|C|^{2}\right) J+\mathcal{Q},
\end{gathered}
$$

then the solutions of the system (3), (11), (15) with $\mathcal{R}=$ $K J$ asymptotically converge to a vicinity of $x^{d}$ :

$$
\begin{aligned}
\limsup _{t \rightarrow+\infty}|x(t)| \leq & \sqrt{\frac{\lambda_{\max }\left(J^{-1}\right)}{\lambda_{\min }\left(J^{-1}\right) \lambda_{\min }(Q)}}(\sqrt{\gamma}|\zeta|+ \\
& \sqrt{2} \max \{|\underline{v}|,|\bar{v}|\}) .
\end{aligned}
$$

The expression of $\mathcal{Z}$ is linear in $J, \mathcal{Q}$ and $\mathcal{R}$. Hence, one can solve (16) as an LMI with respect to these variables by using standard numerical solvers. The control gain can be deduced as follows $K=\mathcal{R} J^{-1}$.

\section{NUMERICAL EXPERIMENTS}

We illustrate the performance of the proposed methodology with a simulated example on the tremor of a Parkinson's disease patient under DBS with constant angular frequency $\omega^{2}(t)=1400, \forall t \geq 0$ and the stimulation amplitude nominal value $a=1$ Volt [14]. Consider the following values as bounds for these parameter values: $\underline{\omega}^{2}=1300, \bar{\omega}^{2}=500 \pi, \underline{a}=0.8$ Volt and $\bar{a}=1.2$ Volt. The measurements of the tremor signal are corrupted by a noise sequence $v(t)$ that consists of independent and identically distributed random numbers sampled from a uniform distribution over the interval $[-V, V]$, with $V=$ 0.1 Volt. The state's initial condition is given by $x(0)=$ $\left[\begin{array}{ll}0.5 & 0.5\end{array}\right]^{\mathrm{T}}$. The measurement of tremor $y$ oscillates from 


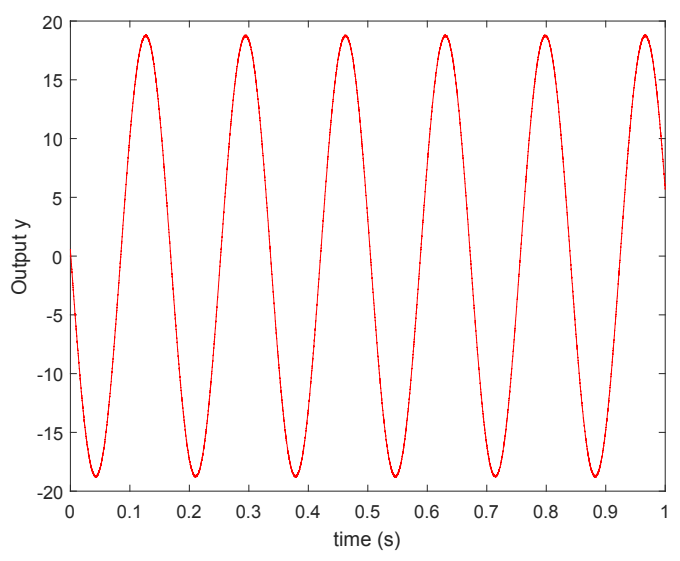

Fig. 1. Evolution of the measurement of the tremor when $u=0$.

-18.8 Volts to 18.8 Volts (Fig. 1), hence it is required to stabilize the system (3). To design the bounds $\underline{x}, \bar{x}$, we select $\underline{x}(0)=\left[\begin{array}{ll}0.4 & 0.4\end{array}\right]^{\mathrm{T}}$ and $\bar{x}(0)=\left[\begin{array}{ll}0.6 & 0.6\end{array}\right]^{\mathrm{T}}$.

We compute the observer gain $L$ as solution of a linear program by using [23, Theorem 7] to ensure boundedness for the estimation errors $\bar{e}$ and $\underline{e}$. We obtain $L=$ $\left[\begin{array}{ll}86963 & 0\end{array}\right]^{\mathrm{T}}$ and the matrix

$$
A_{0}-L C=\left[\begin{array}{cc}
-86963 & 1 \\
1 & 0
\end{array}\right]
$$

is Metzler. It can be inferred from the results of the interval estimation (Fig. 2) that the bounds provided by (11) respect the inclusion relation (12).

To design the control input $u$, we solve the linear matrix inequality (16) by using the YALMIP toolbox for Matlab [31]. Notice the superiority of our control methodology since the control technique that has been proposed in [18] fails to be applied because these authors have considered only noiseless outputs. The conditions of Theorem 2 are satisfied for the systems (3), (11) and (15) with

$$
\begin{aligned}
& \underline{K}=\left[\begin{array}{ll}
-1.33 \times 10^{5} & 1.36 \times 10^{3}
\end{array}\right], \\
& \bar{K}=\left[\begin{array}{ll}
-1.33 \times 10^{5} & 1.7 \times 10^{3}
\end{array}\right] .
\end{aligned}
$$

We see on Fig. 3 that the solution $x_{1}$ (the tremor) of the systems (3), (11) and (15) asymptotically converge to a vicinity of 0 after applying the proposed control methodology, as required.

\section{CONCLUSion}

We have considered the problem of reducing tremor in patients, in the case in which only sets of admissible values are available for the model's disturbances and uncertainties. We have designed a state feedback control to stabilize estimated bounds of the tremor, which are provided by
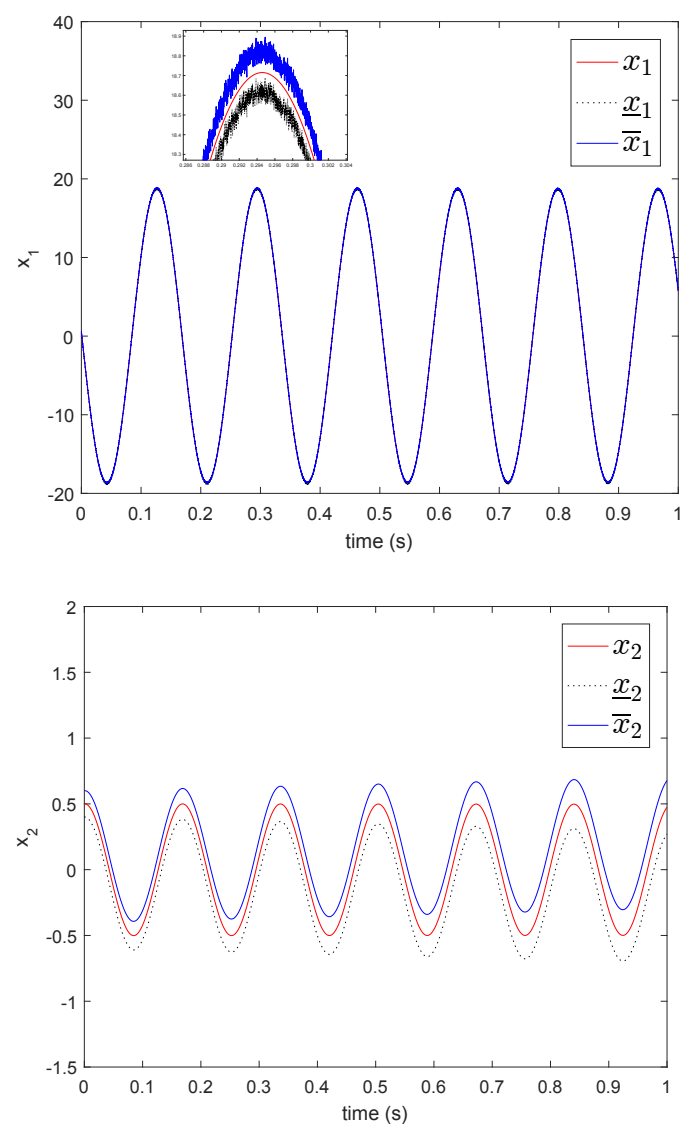

Fig. 2. Evolution of the actual state and the observed bounds without using control methodology $(u=0)$.

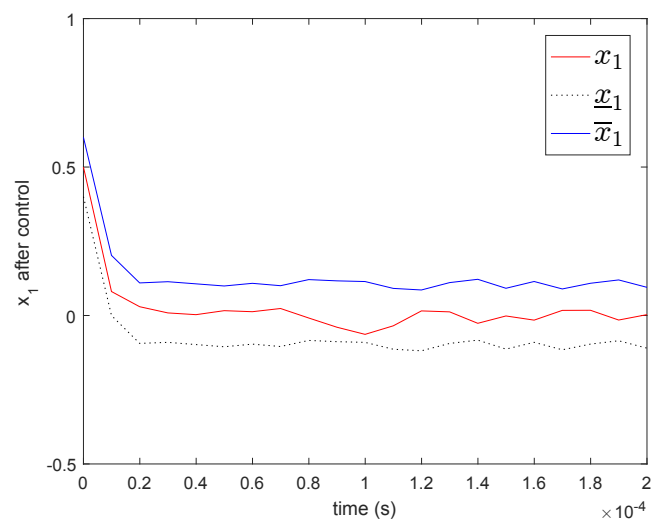

Fig. 3. Evolution of the tremor $\left(x_{1}\right)$ and its estimated bounds $\underline{x}_{1}$ and $\bar{x}_{1}$ with the proposed control methodology. 
an estimator that we have designed. Due to the inclusion relation, this implies the stabilization of the tremor. The performance of our technique is illustrated through numerical simulations. As a direction for future research, the problem of extending this technique to an uncertain oscillator-based nonlinear tremor model can be considered.

\section{REFERENCES}

[1] D. Tarsy, "Handbook of essential tremor and other tremor disorders," Neurology, vol. 70, no. 4, pp. 326-326, 2008.

[2] E. D. Louis and R. Ottman, "How many people in theUSA have Essential Tremor? Deriving a population estimate based on epidemiological data," Tremor and other hyperkinetic movements, vol. 4, no. 259, Aug. 2014.

[3] A. Padilha Lanari Bó, C. Azevedo-Coste, P. Poignet, C. Geny, and C. Fattal, "On the use of FES to attenuate tremor by modulating joint impedance," in Proceedings of the 50th IEEE Conference on Decision and Control and European Control Conference, Orlando, FL, USA, Dec. 2011, pp. 6498-6503.

[4] P. Perozzo, M. Rizzone, B. Bergamasco, L. Castelli, M. Lanotte, A. Tavella, E. Torre, and L. Lopiano, "Deep brain stimulation of the subthalamic nucleus in Parkinson's disease: comparison of preand postoperative neuropsychological evaluation," Journal of the Neurological Sciences, vol. 192, no. 1, pp. 9-15, Nov. 2001.

[5] S. J. Groiss, L. Wojtecki, M. Südmeyer, and A. Schnitzler, "Deep brain stimulation in parkinson's disease," Therapeutic advances in neurological disorders, vol. 2, no. 6, pp. 20-28, Nov. 2009.

[6] J. L. Ostrem and P. A. Starr, "Treatment of dystonia with deep brain stimulation," Neurotherapeutics, vol. 5, no. 2, pp. 320-330, Apr 2008.

[7] L. W. et al., "Modulation of human time processing by subthalamic deep brain stimulation," PLoS One, vol. 6, no. 9, Sep. 2011.

[8] A. M. F. et al., "Reversing cognitive-motor impairments in parkinson's disease patients using a computational modelling approach to deep brain stimulation programming," Brain, vol. 133, no. 3, pp. 746-761, Mar. 2010.

[9] M. Rehan and K.-S. Hong, "Modeling and automatic feedback control of tremor: Adaptive estimation of deep brain stimulation," PLOS ONE, vol. 8, no. 4, pp. 1-11, Apr. 2013.

[10] K. Rouhollahi, M. Emadi Andani, S. M. Karbassi, and I. Izadi, "Designing a robust backstepping controller for rehabilitation in parkinson's disease: a simulation study," IET Systems Biology, vol. 10, no. 4, pp. 136-146, Jul. 2016.

[11] —, "Design of robust adaptive controller and feedback error learning for rehabilitation in parkinson's disease: a simulation study," IET Systems Biology, vol. 11, no. 1, pp. 19-29, Mar. 2017.

[12] K. Rouhollahi, M. Emadi Andani, I. Izadi, and S. M. Karbassi, "Controllability and observability analysis of basal ganglia model and feedback linearisation control," IET Systems Biology, vol. 11, no. 5, pp. 144-154, Oct. 2017.

[13] J. P. Giuffrida, D. E. Riley, B. N. Maddux, and D. A. Heldman, "Clinically deployable Kinesia technology for automated tremor assessment," Movement Disorders, vol. 24, no. 5, pp. 723-730, Apr. 2009.

[14] T. Mera, J. L. Vitek, J. L. Alberts, and J. P. Giuffrida, "Kinematic optimization of deep brain stimulation across multiple motor symptoms in parkinson's disease," Journal of Neuroscience Methods, vol. 198, no. 2, pp. 280-286, Jun. 2011.

[15] F. Mazenc and O. Bernard, "Asymptotically stable interval observers for planar systems with complex poles," IEEE Transactions on Automatic Control, vol. 55, no. 2, pp. 523-527, 2010.

[16] _- "Interval observers for linear time-invariant systems with disturbances," Automatica, vol. 47, no. 1, pp. 140-147, 2011.

[17] D. Efimov, T. Raïssi, S. Chebotarev, and A. Zolghadri, "Interval state observer for nonlinear time-varying systems," Automatica, vol. 49, no. 1 , pp. 200-205, 2013.
[18] D. Efimov, T. Raïssi, and A. Zolghadri, "Control of nonlinear and LPV systems: interval observer-based framework," IEEE Transactions on Automatic Control, vol. 58, no. 3, pp. 773-782, 2013.

[19] K. H. Degue, D. Efimov, and J.-P. Richard, "Stabilization of linear impulsive systems under dwell-time constraints: Interval observerbased framework," European Journal of Control, vol. 42, pp. 1-14, Jul. 2018.

[20] K. H. Degue and J. Le Ny, "Estimation and outbreak detection with interval observers for uncertain discrete-time SEIR epidemic models," International Journal of Control, Jul. 2019.

[21] _ _ "An interval observer for discrete-time SEIR epidemic models," in Proceedings of the American Control Conference (ACC), Milwaukee, WI, USA, Jun. 2018.

[22] K. H. Degue, D. Efimov, and A. Iggidr, "Interval estimation of sequestered infected erythrocytes in malaria patients," in Proceedings of the 15th European Control Conference (ECC), Aalborg, Denmark, June 2016.

[23] S. Chebotarev, D. Efimov, T. Raïssi, and A. Zolghadri, "Interval observers for continuous-time LPV systems with $L_{1} / L_{2}$ performance," Automatica, vol. 58, no. 8, pp. 82-89, 2015.

[24] M. Aqil, K.-S. Hong, M.-Y. Jeong, and S. S. Ge, "Detection of event-related hemodynamic response to neuroactivation by dynamic modeling of brain activity," NeuroImage, vol. 63, no. 1, pp. $553-$ 568 , Oct. 2012.

[25] M. Haeri, Y. Sarbaz, and S. Gharibzadeh, "Modeling the Parkinson's tremor and its treatments," Journal of Theoretical Biology, vol. 236, no. 3, pp. 311-322, Oct. 2005

[26] A. Beuter and M. S. Titcombe, "Modulation of tremor amplitude during deep brain stimulation at different frequencies," Brain and Cognition, vol. 53, no. 2, pp. 190 - 192, Nov. 2003.

[27] G. M. Earhart, M. Hong, S. D. Tabbal, and J. S. Perlmutter, "Effects of thalamic stimulation frequency on intention and postural tremor," Experimental Neurology, vol. 208, no. 2, pp. 257- 263, Dec. 2007.

[28] D. Efimov, L. Fridman, T. Raïssi, A. Zolghadri, and R. Seydou, "Interval estimation for LPV systems applying high order sliding mode techniques," Automatica, vol. 48, pp. 2365-2371, 2012.

[29] L. Farina and S. Rinaldi, Positive Linear Systems: Theory and Applications. New York: Wiley, 2000.

[30] H. Smith, Monotone Dynamical Systems: An Introduction to the Theory of Competitive and Cooperative Systems, ser. Surveys and Monographs. Providence: AMS, 1995, vol. 41

[31] J. Löfberg, "Automatic robust convex programming," Optimization methods and software, vol. 27, no. 1, pp. 115-129, 2012. 